\title{
MODEL THEORY FOR THE HIGHER ORDER PREDICATE CALCULUS
}

\author{
BY \\ STEVEN OREY
}

The theory of models for elementary (often called "first order") axiom systems has grown considerably in the last years. This theory has been developed both for its own interest and as a tool for algebra. There is no corresponding development for model theory for higher order systems. One important reason for this is that much of the first order theory is based on the Gödel completeness theorem or extensions of this theorem guaranteeing the existence of models for consistent sets of formulas. For consistent sets of higher order formulas there need be no models; the Henkin completeness theorem of [2] merely assures the existence of general models.

There are, however, formulas $\phi$ which we call (strongly) standard [with respect to the set of formulas $\mathrm{H}$ ] such that if $M_{1}$ is a general model [for $\mathrm{H}$ ] and $M_{2}$ is a (general) model [for $\mathrm{H}$ ] and if $M_{1}$ and $M_{2}$ have the same ground class (and the higher order universes of $M_{2}$ include those of $M_{1}$ ) then either $M_{1}$ is not a general model for $\phi$ or $M_{2}$ is a (general) model for $\phi$. All elementary formulas are strongly standard; on the other hand for standard (a fortiori for strongly standard) formulas much of the elementary theory carries over. The main problem we consider is the syntactic characterization of (strongly) standard formulas $\left({ }^{1}\right)$.

We call two closed formulas (logically) equivalent [relative to $\mathrm{H}$ ] if every (general) model [for $\mathrm{H}$ ] is a general model for both or neither. From Theorem I we obtain a sufficient syntactic condition for a formula to be strongly standard. It follows from Theorems II and III that if $\mathrm{H}$ is any finite (or infinite) set of formulas $\phi$ is (strongly) standard relative to $\mathrm{H}$ only if it is (logically) equivalent relative to $\mathrm{H}$ (and the union of a certain set of formulas $\Phi_{\phi, \mathrm{H}}$ ) to a formula having the syntactic property referred to in the preceding sentence. Since logical equivalence can be defined syntactically, as was shown in [2], we have syntactic conditions which are sufficient for (relative) strong standardness. For a large class of $\mathrm{H}$ we obtain necessary and sufficient syntactic conditions for $\phi$ to be strongly standard relative to $\mathrm{H}$. For finite $\mathrm{H}$ we show that any formula standard relative to $\mathrm{H}$ must be equivalent relative to $\mathrm{H}$

Presented to the Society, August 24, 1956 under the title Regular classes of relational systems; received by the editors January 23, 1958.

(1) That formulas obtained from elementary ones by prefixing existential quantifiers are standard was observed and made use of by Henkin in [3]. In [5] Mostowski discusses a semantic property closely related to properties considered in this paper. 
to a formula with the syntactic property in question; of course the relation of equivalence is not syntactically definable.

In $\S 2$ some related results on model theory are presented. Applications of standard formulas are indicated.

1. We now describe the version of the predicate calculus we shall deal with. The set of type symbols, $\mathrm{T}$, is the intersection of all $\mathrm{T}^{\prime}$ such that $\iota \in \mathrm{T}^{\prime}$, and if $\tau_{1}, \cdots, \tau_{n} \in \mathrm{T}^{\prime}$ then $\left(\tau_{1} \cdots \tau_{n}\right) \in \mathrm{T}^{\prime}$, where $n=1,2, \cdots$. The rank of $\iota$ is zero, the rank of $\left(\tau_{1} \cdots \tau_{n}\right)$ is $n$; the order of $\iota$ is zero, the order of $\left(\tau_{1} \cdots \tau_{n}\right)$ is one more than the maximum of the orders of $\tau_{i}, 1 \leqq i \leqq n$.

For every natural number $n$ and every $\tau \in \mathrm{T}, X^{\tau, n}$ is a variable of type $\tau$. (We shall use $X, Y, Z$ to represent variables of arbitrary type; the same letters with subscripts will be used to represent variables of the type indicated by the subscript.)

Let $A$ be any function on $\mathrm{T}$ such that for distinct $\tau, \tau^{\prime} \in \mathrm{T} A(\tau)$ and $A\left(\tau^{\prime}\right)$ are disjoint sets, and the range of $A$ contains no variables. $A(\tau)$ will serve as the set of object symbols of type $\tau$ for the system $F_{A}$ which we now describe.

A constant of type $\tau$ is a member of $A(\tau)$. A term of type $\tau$ is a variable or constant of type $\tau$. If $t$ is a term of type $\tau$ the order of $t$ is the order of $\tau$. A primitive symbol is a term or one of the following list of symbols: " $\vee$ ", " $\wedge$ ", “७”, " $E$ ", "(", ")", ",", "=". A primitive formula is either an expression of the form $t\left(t_{1}, \cdots, t_{n}\right)$, where $t$ is a term of type $\left(\tau_{1} \cdots \tau_{n}\right)$ and $t_{i}$ is a term of type $\tau_{i}, i=1, \cdots, n$, or an expression of the form $s_{1}=s_{2}$, where $s_{1}, s_{2}$ are terms of the same type. The class of formulas is the least class containing all primitive formulas and such that if $\phi_{1}, \phi_{2}$ are in the class and $X$ is a variable then $\phi_{1} \vee \phi_{2}, \phi_{1} \wedge \phi_{2}, 7 \phi_{1},(E X) \phi_{1},(X) \phi_{1}$ are in the class. (We shall of ten use small Greek letters to designate formulas.) We write $\phi_{1} \supset \phi_{2}$ as an abbreviation for $\neg \phi_{1} \vee \phi_{2}$ and $\phi_{1} \equiv \phi_{2}$ as an abbreviation for $\left(\phi_{1} \supset \phi_{2}\right) \wedge\left(\phi_{2} \supset \phi_{1}\right)$. Whenever we use two expressions of the form $\phi(t), \phi(s)$ in the same context we are indicating that these are the expressions resulting from a certain expression by substituting $t$ or $s$, respectively, for the same free variable. We say $\phi$ is a formula if $\phi$ is a formula in some system $F_{A}$. If $\Phi$ is a set of formulas $K \Phi$ is to be the set of all constants appearing in the formulas of $\Phi$. We will not distinguish between formulas and unit classes of formulas, e.g. $K \phi$ will be written for $K\{\phi\}$.

A formula containing no variables of order greater than 0 and no constant of order greater than 1 will be called elementary.

Following Henkin [2] we now introduce general models. A frame is a function $G$ on T such that $G(\iota)$ is a nonvoid set and for all natural numbers $n$ and all $\tau_{1}, \cdots, \tau_{n} \in \mathrm{T}, G\left(\left(\tau_{1} \cdots \tau_{n}\right)\right)$ is a nonvoid subset of the set of all subsets of the cartesian product $G\left(\tau_{1}\right) \times \cdots \times G\left(\tau_{n}\right)$. If for all natural numbers $n$ and all $\tau_{1}, \cdots, \tau_{n} \in \mathrm{T}, G\left(\left(\tau_{1} \cdots \tau_{n}\right)\right)$ equals the set of all subsets of $G\left(\tau_{1}\right) \times \cdots \times G\left(\tau_{n}\right) G$ is a standard frame. $G_{1}<G_{2}$ is to hold if and only if $G_{1}(\iota)=G_{2}(\iota)$ and $G_{1}(\tau) \subseteq G_{2}(\tau)$ for all $\tau \in \mathrm{T}$. 
If $G$ is a frame, a function $\pi$ is a $G$-structure if and only if (1) the domain of $\pi$ is a set of constants of some $F_{A},(2)$ if $a \in A(\tau)$ and in the domain of $\pi \pi(a)$ $\in G(\tau) . \pi$ is a structure if and only if there is a frame such that $\pi$ is a $G$-structure.

If $G$ is a frame a $G$-assignment is a function $\nu$ whose domain is the set of all variables and which satisfies $\nu(X) \in G(\tau)$ if $\tau$ is the type of $X$.

An ordered pair $(G, \pi)$ such that $G$ is a frame and $\pi$ a $G$-structure is a general model, or, for the sake of brevity a $g$-model; a $g$-model $(G, \pi)$ such that $G$ is a standard frame is a model. If $\left(G^{\prime}, \pi^{\prime}\right),(G, \pi)$ are two $g$-models $\left(G^{\prime}, \pi^{\prime}\right)$ $<(G, \pi)$ is to hold just in case $G^{\prime}<G$ and $\pi^{\prime}=\pi$.

A $g$-model $(G, \pi)$ is admissible for a set of formulas $\Phi$ if $K \Phi$ is included in the domain of $\pi$.

Let $\phi$ be a formula, $(G, \pi)$ a $g$-model admissible for $\phi, \nu$ a $G$-assignment. $\nu$ satisfies $\phi$ in $(G, \pi)$ is defined by induction on the number of logical operators occurring in $\phi$. For this purpose we introduce the function $\pi_{\nu}$ agreeing with $\pi$ on $K \phi$ and with $\nu$ on all variables.

(1) If $\phi$ is $t\left(t_{1}, \cdots, t_{n}\right) \nu$ satisfies $\phi$ if and only if $\pi_{\nu}(t)\left(\pi_{\nu}\left(t_{1}\right), \cdots, \pi_{\nu}\left(t_{n}\right)\right)$ holds;

(2) if $\phi$ is $t_{1}=t_{2} \nu$ satisfies $\phi$ if and only if $\pi_{\nu}\left(t_{1}\right)$ is identical with $\pi_{\nu}\left(t_{2}\right)$;

(3) if $\phi$ is not primitive $\nu$ satisfies $\phi$ if and only if it does so according to the usual inductive definition (cf. [2]).

If $\Phi$ is a set of formulas and $(G, \pi)$ an admissible $g$-model $\nu$ satisfies $\Phi$ if and only if it satisfies each formula of $\Phi$. In case all $G$-assignments $\nu$ satisfy $\Phi$ we say $(G, \pi)$ is a $g$-model for $\Phi$ or $\Phi$ holds true in $(G, \pi) . \Phi_{1}$ (logically) implies $\Phi_{2}\left[\right.$ relative to $\mathrm{H}$ ] if for every (general) model admissible for $\Phi_{1}$ and $\Phi_{2}$ [which is a $g$-model for $\mathbf{H}$ ] every assignment satisfying $\Phi_{1}$ satisfies $\Phi_{2}$. Then (logical) equivalence [relative to $\mathrm{H}$ ] is defined as the corresponding two way implication.

A set of closed formulas $\Phi$ will be called (strongly) standard [relative to $\mathrm{H}$ ] if and only if for every general model $\left(G^{\prime}, \pi\right)$ for $\Phi$ [in which $\mathrm{H}$ holds] and every (general) model $(G, \pi)$ [in which $\mathrm{H}$ holds] such that $G^{\prime}<G,(G, \pi)$ is a (general) model for $\Phi$.

If $\Phi$ is standard and consistent it has $g$-models by [2], and therefore models. In fact much of the theory of elementary classes carries over to give more detailed information about models for $\Phi$. Every set of elementary formulas is of course actually strongly standard.

It is obvious that the relativized concepts will be of interest. In particular one may be interested in the case where $\mathrm{H}$ is some set of axioms for a class of algebraic systems; also the case where $\mathrm{H}$ is a set of axioms for set theory is of interest. One may wish to take for $\mathrm{H}$, for example, all instances of the comprehension axiom; note that for this important case our results completely solve the problem of characterizing strongly standard formulas relative to $\mathrm{H}$.

For single formulas the concepts dual to those above can be introduced by defining a closed formula $\phi$ to be (strongly) standard decreasing [relative 
to $\mathrm{H}$ ] just in case $\rceil \phi$ is (strongly) standard [relative to $\mathrm{H}$ ]. We proceed to the problem of characterizing (strongly) standard decreasing formulas; obviously the dual problem is solved by dualizing the solution.

In connection with the following definitions it is important to remember that $\supset$ and $\equiv$ are not primitive symbols of our formalism.

$\phi$ is 7 -simple if no higher order quantifier occurs in the scope of a 7 symbol. $\phi$ is in normal form if it is a closed formula in prenex form with the matrix in disjunctive normal form.

Let $\dot{t}, \dot{X}$ stand for particular occurrences of a term $t$ and a variable $X$ in a formula $\phi$. We say $\dot{t}$ immediately secures $\dot{X}$ in $\phi$ if and only if

(1) $\phi$ is $\dot{t}=\dot{X}$ or $\dot{X}=\dot{t}$ or $\dot{t}(\cdots, \dot{X}, \cdots)$, or

(2) $\phi$ is $\phi_{1}(\ddot{X}, \dot{t}) \wedge \phi_{2}(\dot{X})$ or $\phi_{2}(\dot{X}) \wedge \phi_{1}(\ddot{X}, \dot{t}), \ddot{X}, \dot{X}$ being free occurrences of $X$ in $\phi_{1}, \phi_{2}$ respectively, $\dot{i}$ immediately secures $\ddot{X}$ in $\phi_{1}$, and $\vee$ does not occur in $\phi_{1}$, or

(3) $i$ immediately secures $\dot{X}$ in $\phi_{1}$ and $\phi_{1}$ is a subformula of $\phi$ such that $\phi_{1}$ is not in the scope of a 7 -sign.

A sequence of occurrences $\left(\dot{X}, \dot{t}_{1}, \cdots, \dot{t}_{n}\right)$ will be called a securing chain in $\phi$ of $\dot{X}$ if

(a) $\dot{t}_{1}$ immediately secures $\dot{X}$ in $\phi$,

(b) $\dot{t}_{i+1}$ immediately secures $\dot{t}_{i}$ in $\phi$,

(c) $i_{n}$ is an occurrence of a constant or a variable bound by a universal quantifier.

The number $n$ is the length of the securing chain. When (a)-(c) hold $\dot{t}_{n}$ secures $\dot{X}$. A minimal securing chain for $\dot{X}$ is a securing chain for $\dot{X}$ of minimal length. $\dot{X}$ (immediately) depends on $\dot{Y}$ if there is a minimal securing chain for $\dot{X}$ in which $\dot{Y}$ occurs (as first component after $\dot{X}$ ).

An occurrence of a variable in a formula will be called proper if it is not part of a quantifier. A formula $\phi$ is secure if it is closed and 7 -simple and for every proper occurrence of a higher order variable in $\phi$ which is existentially bound there exists a securing chain in $\phi$ of that occurrence. $\phi$ is order secure if $\phi$ is secure and, if $(E \dot{X}),(E \dot{Y})$ are occurrences of higher order quantifiers, then either no occurrence of $X$ bound by the first quantifier depends on an occurrence of $Y$ bound by the second quantifier or no occurrence of $Y$ bound by the second quantifier depends on any occurrence of $X$ bound by the first quantifier.

Lemma. $A$ secure formula $\phi$ is logically equivalent to an order secure formula in normal form.

Proof. It is easy to check that any secure formula is logically equivalent to a secure formula in normal form if one notes the following fact: If $i$ secures $\dot{X}$ in $\phi$ and $\dot{t}$ occurs in a subformula $\gamma$ of $\phi$, then $\gamma$ is not in the scope of a 7 -symbol.

Now let $\phi$ be a secure formula in normal form and let $p$ be the number of 
disjunctive components in the matrix of $\phi$. Let $X_{1}, \cdots, X_{t}$ be a listing of all higher order variables occurring existentially quantified in the prefix of $\phi$, ordered in such a way that for $1 \leqq i<j \leqq t$ the order of $X_{i}$ is not less than that of $X_{j}$. If $\phi$ is not order secure let $(v, i, j)$ be the lexicographically first triple satisfying $1 \leqq v \leqq p, 1 \leqq i<j \leqq t$, and some occurrence of $X_{i}$ in the vth disjunctive component of $\phi$ immediately depends on some occurrence of $X_{j}$ in the $v$ th disjunctive component of $\phi$. Note that the condition on $(v, i, j)$ implies that $X_{i}=X_{j}$ or $X_{j}=X_{i}$ occurs un-negated in the $v$ th disjunctive component of $\phi$. Modify $\phi$ by interchanging all occurrences of $X_{i}$ and $X_{j}$ in the $v$ th disjunctive component; this leads to a logically equivalent formula. Repeating this process a finite number of times the desired order secure formula is obtained; to see this one needs only to check that at each step after the first one the relevant $(v, i, j)$ lexicographically follows the $(v, i, j)$ used at the preceding step.

We now explain some notions that will be useful in the proof of Theorem I. Let $\psi$ be a quantifier free formula in which the free variables are $X_{1}, \cdots$, $X_{4}$ in order of appearance from left to right. Let $\tau_{i}$ be the type of $X_{i}, i$ $=1, \cdots, 4$. Then each $g$-model $(G, \pi)$ admissible for $\psi$ associates with this formula a truth function $\psi^{*}\left(\xi_{1}, \cdots, \xi_{4}\right)$, where $\xi_{i}$ is a variable ranging over $G\left(\tau_{i}\right), i=1, \cdots, 4$, and $\psi^{*}\left(n_{1}, \cdots, n_{4}\right)$ is true (false) if the assignments which assign $n_{i}$ to $X_{i}, i=1, \cdots, 4$, satisfy (do not satisfy) $\psi$. If $\phi$ is the formula $\left(E X_{1}\right)\left(X_{2}\right)\left(X_{3}\right)\left(E X_{4}\right) \psi$ there is associated by $(G, \pi)$ a truth-functional $\phi^{*}\left(\xi_{2}, \xi_{3} ; \eta_{1}, \eta_{4}\right)$, where $\xi_{2}, \xi_{3}$ are as above, $\eta_{1}$ is a variable ranging over functions of zero arguments with values in $G\left(\tau_{1}\right)$, and $\eta_{4}$ is a variable ranging over functions from $G\left(\tau_{2}\right) \times G\left(\tau_{3}\right)$ into $G\left(\tau_{4}\right)$ and $\phi^{*}\left(n_{2}, n_{3} ; f_{1}, f_{4}\right)=\psi^{*}\left(f_{1}, n_{2}, n_{3}\right.$, $f_{4}\left(n_{2}, n_{3}\right)$ ). Similarly truth functions are associated with arbitrary quantifier free formulas and truth functionals with arbitrary closed formulas in prenex form. If for some truth functional $\phi^{*}\left(\xi_{1}, \cdots, \xi_{s} ; f_{1}, \cdots, f_{t}\right)$ is true for all values of $\xi_{1}, \cdots, \xi_{s}$ in some $g$-model we say $f_{1}, \cdots, f_{t}$ verify $\phi^{*}$ in that $g$ model.

Theorem I. If $\phi$ is logically equivalent (relative to $\mathrm{H}$ ) to a secure formula, $\phi$ is strongly standard decreasing (relative to $\mathrm{H}$ ).

Proof. Let $\phi$ be an order secure formula in normal form. In view of the lemma, Theorem I will follow from a proof that $\phi$ is strongly standard decreasing.

Let $X_{1}, \cdots, X_{t}$ be a list of all existentially quantified variables occurring in $\phi$, ordered in such a way that for $1 \leqq i<j \leqq t$ it is never true that $X_{i}$ is a higher order variable such that some occurrence of $X_{i}$ depends on some occurrence of $X_{j}$. Let $\phi^{*}=\phi^{* *}\left(\bar{\xi}_{;} ; \eta_{1}, \cdots, \eta_{t}\right)$, where $\bar{\xi}$ is the vector made up of the universal arguments of $\phi$, and $\eta_{i}$ is the argument corresponding to $X_{i}$.

Let $\left(G^{\prime}, \pi\right)<(G, \pi)$ and assume $f_{1}, \cdots, f_{t}$ verify $\phi^{*}$ in $(G, \pi)\left({ }^{2}\right)$. We show

(2) The functional $\phi^{*}$ depends on the model $(G, \pi)$. Instead of exhibiting this dependence explicitly, say by a subscript, we use phrases such as "the value of $\phi^{*}$ in $(G, \pi)$. " 
how to choose $g_{1}, \cdots, g_{t}$ verifying $\phi^{*}$ in $\left(G^{\prime}, \pi\right)$. Let $\bar{n}$ be a value of $\bar{\xi}$ with all components in $G^{\prime}$ (i.e., in the range of $G^{\prime}$ ). Let $\bar{n}_{1}, \cdots, \bar{n}_{t}$ be the subvectors of $\bar{n}$ appearing as arguments of $f_{1}, \cdots, f_{t}$ respectively, when $\phi^{* *}\left(\bar{n} ; f_{1}, \cdots, f_{t}\right)$ is being evaluated. Let $g_{1}, \cdots, g_{t}$ be functions of a suitable type such that $(1) g_{i}\left(\bar{n}_{i}\right)$ is in $G^{\prime}, i=1, \cdots, t ;(2)$ if $f_{i}\left(\bar{n}_{i}\right)$ is in $G^{\prime}, g_{i}\left(\bar{n}_{i}\right)$ $=f_{i}\left(\bar{n}_{i}\right)$. We shall show $\phi^{* *}\left(\bar{n} ; g_{1}, \cdots, g_{t}\right)$ is true; so by choosing $g_{1}, \cdots, g_{t}$ so as to satisfy (1) and (2) for each $\bar{n}$ in $G^{\prime}$ functions verifying $\phi^{* *}$ in $\left(G^{\prime}, \pi\right)$ are obtained.

Assuming now that $g_{1}, \cdots, g_{t}$ satisfy (1) and (2) and choosing $\bar{n}$ in $G^{\prime}$ we prove by induction that $\phi^{* *}\left(\bar{n} ; g_{1}, \cdots, g_{i}, f_{i+1}, \cdots, f_{t}\right)$ is true in $(G, \pi)$ for $i=0, \cdots, t$. For $i=t$ this is the desired conclusion, for $i=0$ it follows from the assumption that $f_{1}, \cdots, f_{t}$ verify $\phi^{*}$ in $(G, \pi)$. So suppose the assertion holds for $i \leqq k$ and consider $\phi^{* *}\left(\bar{n} ; g_{1}, \cdots, g_{k}, g_{k+1}, f_{k+2}, \cdots, f_{t}\right)$.

CASE 1. $f_{k+1}\left(\bar{n}_{k+1}\right)$ is in $G^{\prime}$. Then $g_{k+1}\left(\bar{n}_{k+1}\right)=f_{k+1}\left(\bar{n}_{k+1}\right)$ and the assertion reduces to that for $i=k$.

CASE 2. $f_{k+1}\left(\bar{n}_{k+1}\right)$ is not in $G^{\prime}$. Assume $\phi^{* *}\left(\bar{n} ; g_{1}, \cdots, g_{k}, g_{k+1}, f_{k+2}, \cdots, f_{t}\right)$ does not hold; we shall obtain a contradiction. For it now follows that there is a disjunctive component $\gamma$ of the matrix of $\phi$ with the following properties. $X_{k+1}$ occurs in $\gamma$. And if $\gamma^{* *}$ is the truth function associated with $\gamma$ (considered as a function of all variables occurring in the truth function of the matrix of $\phi$ and with the order of arguments as indicated by the succeeding formulas), $\gamma^{* *}\left(\bar{n}, g_{1}\left(\bar{n}_{1}\right), \cdots, g_{k}\left(\bar{n}_{k}\right), f_{k+1}\left(\bar{n}_{k+1}\right), \cdots, f_{t}\left(\bar{n}_{t}\right)\right)$ is true but $\gamma^{* *}\left(\bar{n}, g_{1}\left(\bar{n}_{1}\right)\right.$, $\left.\cdots, g_{k+1}\left(\bar{n}_{k+1}\right), f_{k+2}\left(\bar{n}_{k+2}\right), \cdots, f_{t}\left(\bar{n}_{t}\right)\right)$ is false. Since $X_{k+1}$ is secured, $\gamma$ must have a conjunctive component of the form $t=X_{k+1}$ or $X_{k+1}=t$ or

$$
t\left(\cdots, X_{k+1}, \cdots\right) \text {, }
$$

where $t$ is a constant or a universally quantified variable or an existentially bound variable with an occurrence on which depends an occurrence of $X_{k+1}$ in $\phi$. Thus either $t^{*}=f_{k+1}\left(\bar{n}_{k+1}\right)$ or $t^{*}\left(\cdots, f_{k+1}\left(\bar{n}_{k+1}\right), \cdots\right)$, where $t^{*}=\pi(a)$, $a \in K \phi$, or $t^{*}$ is a component of $\bar{n}$ or $t^{*}=g_{j}\left(\bar{n}_{j}\right), 1 \leqq j \leqq k$. In all cases $t^{*}$, and therefore $f_{k+1}\left(\bar{n}_{k+1}\right)$ is in $G^{\prime}$, contradicting the assumption that we are in Case 2. This completes the proof of the theorem.

We now search for necessary conditions for a formula to be (strongly) standard decreasing relative to some given set of formulas.

For the remainder of the discussion $Y_{(\tau)}$ is to be some definite variable of type $(\tau)$, for each type symbol $\tau$ other than $\iota$.

Let $\tau_{1}, \cdots, \tau_{n}$ be a finite sequence of type symbols, and let $\tau_{1}^{\prime}, \cdots, \tau_{m}^{\prime}$ be the subsequence obtained by dropping from the original sequence all elements equal to $\iota$. Let $\tau=\left(\tau_{1} \cdots \tau_{n}\right)$. We define $\alpha_{\tau}$ to be the following formula: $\left(E X_{\tau}\right) Y_{(\tau)}\left(X_{\tau}\right) \wedge\left(X_{\tau}\right)\left(Z_{\tau_{1}}\right) \cdots\left(Z_{\tau_{n}}\right)\left(\left(Y_{(\tau)}\left(X_{\tau}\right) \wedge X_{\tau}\left(Z_{\tau_{1}}, \cdots, \quad Z_{\tau_{n}}\right)\right)\right.$ $\left.\supset\left(Y_{\left(\tau_{1}\right)}^{\prime}\left(Z_{\tau_{1}}^{\prime}\right) \wedge \cdots \wedge Y_{\left(\tau_{m}\right)}^{\prime}\left(Z_{\tau_{m}}^{\prime}\right)\right)\right)$.

Let $\phi$ be a closed formula and $\mathrm{H}$ a set of closed formulas. Assume none of the $Y_{(\tau)}$ occur in $\phi$ or any formula of $\mathrm{H}$. Let $\mathrm{T}_{\phi, \mathrm{H}}^{\prime}$ be the least set of type sym- 
bols such that (1) $\tau$ is in the set if some term of type $\tau$ occurs in $\phi$ or in some formula of $\mathrm{H},(2)$ if $\left(\tau_{1} \cdots \tau_{n}\right)$ is in the set so are $\tau_{1}, \cdots, \tau_{n} . \mathrm{T}_{\phi, \mathrm{H}}$ is to be $\mathrm{T}_{\phi, \mathrm{B}}-\{\iota\}$.

If $\gamma$ is any closed formula $\gamma_{R}$ is to be the formula obtained by restricting every variable of type $\tau$ to $Y_{(\tau)}$ for $\tau \neq \imath$; i.e. occurrences of $\left(X_{\tau}\right) \psi,\left(E X_{\tau}\right) \psi$ are replaced by $\left(X_{\tau}\right)\left(Y_{(\tau)}\left(X_{\tau}\right) \supset \psi\right),\left(E X_{\tau}\right)\left(Y_{(\tau)}\left(X_{\tau}\right) \wedge \psi\right)$ respectively. We write $\{\phi ; H\}$ for the least set of formulas such that (1) $\alpha_{\tau}$ is in the set for every $\tau \in \mathrm{T}_{\phi, \mathrm{H}}$, (2) $\eta_{R}$ is in the set for every $\eta \in \mathrm{H}$, (3) if $a$ is a constant of type $\tau, \tau \neq \iota, a \in K H \cup K \phi$ then $Y_{(\tau)}(a)$ is in the set, (4) $\rceil \phi_{R}$ is in the set. In case $\mathrm{H}$ is finite $\{\phi ; \mathrm{H}\}$ is finite, and $[\phi, \mathrm{H}]$ is to stand for the conjunction in some order of all formulas of $\{\phi ; \mathrm{H}\}$. By $(\bar{Y})\rceil[\phi, \mathrm{H}]$ we denote the universal closure of $\neg[\phi, \mathrm{H}]$. It is easy to verify that $(\bar{Y})\rceil[\phi, \mathrm{H}]$ is logically equivalent to a secure formula.

Let $\boldsymbol{\Xi}_{\phi, \mathrm{H}}$ be the set of formulas $\left\{\left(E X_{(\tau)}\right)\left(X_{\tau}\right) X_{(\tau)}\left(X_{\tau}\right)\right\}, \tau \in \mathbf{T}_{\phi, \mathrm{H}}$.

Theorem II. Let $\phi$ be a closed formula, $\mathrm{H}$ a set of closed formulas and $\mathrm{H}^{\prime}$ a finite subset of $\mathrm{H}$; then $(\bar{Y})\rceil\left[\phi, \mathrm{H}^{\prime}\right]$ (logically) implies $\phi$ relative to (the union of $\Xi_{\phi, \mathrm{H}}$ and) $\mathrm{H}$.

Proof. Since $\Xi_{\phi, \mathrm{H}}$ holds in every model it will be sufficient to prove the part of the theorem dealing with logical implication. So let $(G, \pi)$ be a $g$ model for $\mathrm{H}, \boldsymbol{\Xi}_{\phi, \mathrm{H}}$ and $\left.(\bar{Y})\right\rceil\left[\phi, \mathrm{H}^{\prime}\right]$. There exist then assignments $\nu$ such that $\nu\left(Y_{(\tau)}\right)=G(\tau)$ for all $\tau \in \mathrm{T}_{\phi, \mathrm{H}}$. These $\nu$ must satisfy $\rceil[\phi, \mathrm{H}]$; but since $\mathrm{H}$ is true in $(G, \pi)$ this is possible only if they satisfy $\phi_{R}$. But these $\nu$ satisfy $\phi_{R}$ if and only if they satisfy $\phi$; it follows that $(G, \pi)$ is a $g$-model for $\phi$.

THEOREM III. Let $\phi$ be a closed formula and $\mathrm{H}$ a finite (or infinite) set of closed formulas. If $\phi$ is (strongly) standard decreasing relative to $\mathrm{H}$ then $\phi$ (logically) implies $(\bar{Y})\rceil\left[\phi, \mathrm{H}^{\prime}\right]$ relative to $\mathrm{H}$ for some finite subset $\mathrm{H}^{\prime}$ of $\mathrm{H}$.

Proof. Let $\phi$ be strongly standard decreasing relative to $\mathrm{H}$. Let $\mathrm{H}^{\prime \prime}$ be obtained from $\mathrm{H}$ by adding $\left(X_{\tau}\right)\left(X_{\tau}=X_{\tau}\right)$ for all $\tau$. Let $(G, \pi)$ be some $g$-model for $\mathrm{H}$ and $\phi$, and suppose there exists an assignment $\nu$ satisfying $\left\{\phi ; \mathrm{H}^{\prime \prime}\right\}$. Letting $G^{\prime}(\iota)=G(\iota)$ and $G^{\prime}(\tau)=\nu\left(Y_{(\tau)}\right)$ for $\tau \neq \iota$ one obtains a $g$-model $\left(G^{\prime}, \pi\right)$ making $\mathrm{H}$ true and $\phi$ false and satisfying $\left(G^{\prime}, \pi\right)<(G, \pi)\left({ }^{3}\right)$. This contradicts the assumption that $\phi$ is strongly standard decreasing relative to $\mathrm{H}$. So $\mathrm{H}, \phi$ and $\left\{\phi, \mathrm{H}^{\prime \prime}\right\}$ cannot be simultaneously satisfied. The completeness theorem of Henkin [2] being available, we can infer that there is a finite subset $\mathrm{H}^{\prime}$ of $\mathbf{H}$ such that $\mathrm{H}, \phi,\left[\phi, \mathrm{H}^{\prime}\right]$ cannot simultaneously be satisfied. In other words $\phi$ implies $(\bar{Y}) \neg\left[\phi, \mathrm{H}^{\prime}\right]$ relative to $\mathrm{H}$, as was to be shown.

A rather similar argument establishes the other part of the theorem.

In case $\mathrm{T}_{\phi, \mathrm{H}}^{\prime}=\mathrm{T}$ it is possible that $\Xi_{\phi, \mathrm{H}}$ holds in every general model in

( $\left.{ }^{3}\right)$ That $\left(G^{\prime}, \pi\right)$ is a $g$-model depends on the fact that for all $\tau$ different from $\iota \alpha_{\tau} \in\left\{\phi ; \mathbf{H}^{\prime \prime}\right\}$; this is the reason for considering $\mathrm{H}^{\prime \prime}$ rather than $\mathrm{H}$. 
which $\mathrm{H}$ holds. Results for this special case and the case $\mathrm{H}$ finite are summarized in the following theorem.

THEOREM IV. Let $\phi$ be a closed formula and $\mathrm{H}$ a finite (or infinite) set of closed formulas (such that $\Xi_{\phi, \mathrm{H}}$ holds in every g-model of $\mathrm{H}$ ). Then the following conditions are equivalent.

(1) $\phi$ is (strongly) standard decreasing relative to $\mathrm{H}$.

(2) There exists a finite subset $\mathrm{H}^{\prime}$ of $\mathrm{H}$ such that $\phi$ is (logically) equivalent to $(\bar{Y})\rceil\left[\phi, \mathrm{H}^{\prime}\right]$ relative to $\mathrm{H}$.

(3) ( $\phi$ is logically equivalent to some secure formula relative to $\mathrm{H}$.)

Note. There are only two equivalent conditions for standard decreasing formulas; a formula $\phi$ can be equivalent to a standard decreasing formula without itself being standard decreasing.

Proof. Theorems II and III immediately give the implications from (1) to (2). Since formulas of the form $(\bar{Y})\rceil\left[\phi, \mathrm{H}^{\prime}\right]$ are logically equivalent to secure formulas by an earlier remark, we have the implication from (2) to (3). The implication of (1) from (3) is Theorem $I$.

It remains to show that if $\phi$ is equivalent to $(\bar{Y})\rceil\left[\phi, \mathbf{H}^{\prime}\right]$ relative to $\mathbf{H}$ then $\phi$ is standard decreasing relative to $H$, where $H^{\prime}$ is any finite subset of $H$. So assume the equivalence and let $(G, \pi)$ be a model for $\phi$ and H. Let $\left(G^{\prime}, \pi\right)$ be a $g$-model for $\mathrm{H}$ such that $\left(G^{\prime}, \pi\right)<(G, \pi)$. Since $G$ is a standard frame there is a $G$-assignment $\nu$ such that $\nu\left(Y_{(\tau)}\right)=G^{\prime}(\tau)$ for all $\tau$ other than $\iota . \nu$ must satisfy $\rceil\left[\phi, \mathrm{H}^{\prime}\right]$; but since $\left(G^{\prime}, \pi\right)$ is a $g$-model for $\mathbf{H}^{\prime}$ this is possible only if $\nu$ satisfies $\phi_{R}$. It follows that $\left(G^{\prime}, \pi\right)$ is a $g$-model for $\phi$.

It may be noted that the proof of the equivalence of (1) and (2) when read without the parenthesized parts is much more straightforward than the corresponding equivalence with the parenthesized parts. Indeed, if $\mathbf{H}$ is finite, the formula $(\bar{Y})\rceil[\phi, \mathrm{H}]$ intuitively just says that $\phi$ is true when the higher order variables are restricted to arbitrary universes satisfying the axioms $\mathbf{H}$; and so if $(\bar{Y})\rceil[\phi, H]$ and $\mathrm{H}$ holds in a model $(G, \pi), \phi$ must indeed hold in every $g$-model $\left(G^{\prime}, \pi\right)$ such that $\left(G^{\prime}, \pi\right)<(G, \pi)$ and $\mathrm{H}$ is true in $\left(G^{\prime}, \pi\right)$. On the other hand if $(\bar{Y})\rceil[\phi, \mathrm{H}]$ and $\mathrm{H}$ hold in some $g$-model $(G, \pi)$ the only thing that seems evident is that $\phi$ holds in every $g$-model $\left(G^{\prime}, \pi\right)$ satisfying $\left(G^{\prime}, \pi\right)<(G, \pi)$, H true in $\left(G^{\prime}, \pi\right)$ and $G^{\prime}(\tau)$ an element of $G((\tau))$ for every $\tau \in \mathrm{T}_{\phi, \mathrm{H}}$. It is of course Theorem I that shows the italicised restriction to be superfluous.

2. If $A$ is a subset of the range of some frame $G$ the trail of $A$ is to be the intersection of all sets $S$ satisfying (1) $A \subseteq S$, and (2) if $v \in S$ and $\left(v_{1}, \cdots, v_{n}\right)$ $\in v$ then $v_{1} \in S, \cdots, v_{n} \in S$.

In case $B$ is a set of constants and $\pi$ a structure we shall write $\pi(B)$ for $\{\pi(b): b \in B\}$.

Consider a formula $\phi$ in prenex form with associated truth functional $\phi^{*}\left(\bar{\xi} ; \eta_{1}, \cdots, \eta_{t}\right)$ such that for every $g$-model $(G, \pi)$ of $\phi$ there exist functions 
$f_{1}, \cdots, f_{t}$ verifying $\phi^{*}$ in $(G, \pi)$ and such that for every value $\bar{n}$ of $\bar{\xi}$ and $i=1, \cdots, t, f_{i}\left(\bar{n}_{i}\right)$ is in the trail of the set consisting of the elements of $\pi(K \phi)$ and the components of $\bar{n}$. Such a $\phi$ is logically equivalent to a secure formula; (there are however secure formulas not enjoying this semantic property). If one could show that every strongly standard decreasing formula is logically equivalent to a formula enjoying the semantic property of the formula $\phi$ one would obtain a proof of the equivalence of (1) and (3) of Theorem IV without the hypothesis involving $\Xi_{\phi, \mathrm{H}}$.

To motivate the following discussion we consider an example. Call a set finite if it is similar to a segment of the natural numbers. It is familiar that the concept of finite set cannot be defined by an elementary formula. Can this concept be defined by a formula all of whose constants and variables are of order at most one? The question is vague, and there are a number of ways of making it precise. One way of making the problem definite which seems natural is to ask whether there exists a formula $\phi$ of the required kind containing a single constant $P$ of type $(\iota)$ such that in every model for $\phi P$ is made to correspond to a finite set, and, conversely, for every finite set $M$ there exists a model for $\phi$ such that $P$ corresponds to $M$. From this point of view $\phi$ defines a class of structures (in the sense in which this word was introduced in \$1) rather than a class of models. We shall be considering classes of structures such that any two structures in a class have the same domain, e.g. the class of structures $\pi$ with domain $\{P\}$, where $P$ is as above and $\pi(P)$ is a finite set. We proceed to some definitions.

Let $C$ be a class of structures with common domain $A$, and $\Phi$ a set of formulas such that (a) every formula in $\Phi$ is closed, (b) $K \Phi=A$. Then $\Phi d e$ fines $C$ if and only if (1) if $(G, \pi)$ is a model for $\Phi$ and the domain of $\pi$ is $A$ then $\pi \in C$, (2) if $\pi \in C$ there exists a model $(G, \pi)$ for $\Phi ; \Phi \alpha$-defines $C$ if and only if (1), and $\left(2^{\prime}\right)$ if $\pi \in C$ and $(G, \pi)$ is a model then $\Phi$ is true in $(G, \pi)$; $\Phi \beta$-defines $C$ if and only if $\left(1^{\prime}\right)$ if $(G, \pi)$ is a model for $\Phi$ and the domain of $\pi$ is $A$, then $\pi \in C$ and $G(\iota)$ is included in the trail of $\pi(A)$, and (2). If $\Phi$ violates (a) or (b) it does not define, $\alpha$-define, or $\beta$-define $C$.

Note that for every structure $\pi$ there is a unique model $(G, \pi)$ such that $G(\iota)$ is included in the trail of the range of $\pi$; in case $\Phi$ holds in this model we shall say $\Phi$ holds in $\pi$.

Clearly if $\phi \alpha$-defines $C$ one can construct $\phi^{\prime} \beta$-defining $C$; also, if $\Phi$ $\alpha$-defines or $\beta$-defines $C$ then $\Phi$ defines $C$.

Let $C$ be a class of structures with common domain; to $C$ assign the class of all models $M$ such that $(G, \pi) \in M$ if and only if $\pi \in C$ and $(G, \pi)$ is the unique model with $G(\iota)$ included in the trail of the range of $\pi$. In the literature expressions such as " $\Phi$ is an axiom system for $M$ " are frequently used for what we would express by " $\Phi \beta$-defines $C$." Perhaps $\beta$-definability is more natural than definability for elementary axiom systems; for higher order systems this does not seem to be the case. In any case the distinction between these notions is not so important for elementary formulas since, according to 
Theorem IV, if $C$ is defined by some elementary formula $\phi$ we can construct an elementary formula $\phi^{\prime} \beta$-defining $C$. On the other hand one obtains the following result by a slight extension of the argument in Hintikka [4]: if $\phi$ is a formula containing no constants of order greater than 1 and $\phi$ defines $C$ one can construct a formula $\phi^{\prime}$ containing no variable of order higher than 1 such that $\phi^{\prime}$ defines $C$. The constructed formula will never $\alpha$-define or $\beta$-define $C$.

From our point of view the significance of Skolem's paradox and other related results is the following: there exist many simple classes of structures $C$ such that all structures in $C$ have a common domain, this domain consisting entirely of constants of order one or zero, which cannot be defined by elementary formulas. Such results are in sharp contrast to the extension of the result of [4] mentioned in the preceding paragraph and bear out Hintikka's point that the step from elementary formulas to formulas involving variables of order 1 is crucial, whereas further steps up in the order hierarchy are relatively less significant.

We now turn to Theorem IV. Note that if $\Phi$ is a set of elementary formulas defining a class $C$ carrying through the construction of the proof of Theorem IV for each $\phi \in \Phi$ separately leads to a set of elementary formulas $\alpha$-defining $C$; on the other hand easy examples show that there need not exist a set of elementary formulas $\beta$-defining $C$.

THeOREM IV. If $\phi$ is an elementary formula defining the class $C$ there can be found an elementary formula $\alpha$-defining $C$, and hence also an elementary formula $\beta$-defining $C$.

Proof. Let $\phi$ be an elementary formula defining $C$. By renaming bound variables in $\phi$ we can obtain a formula in which no variable occurs in more than one quantifier; we shall assume $\phi$ already has this form. It is easy to write down a formula $\Delta(X)$ with $K \Delta(X)=K \phi$ such that an assignment $\nu$ satisfies $\Delta(X)$ in a $g$-model $(G, \pi)$ admissible for $\Delta(X)$ if and only if $\nu(X)$ is an individual in the trail of $\pi(K \Delta(X))$. (In the proof of this theorem all variables mentioned are individual variables.)

Clearly if $\phi^{\prime} \alpha$-defines $C \phi^{\prime} \wedge(X) \Delta(X) \beta$-defines $C$, so that it suffices to prove the first assertion of the theorem.

Let $\phi_{R}$ be the formula obtained from $\phi$ by first replacing all occurrences of the form $(E Z) \theta$ by $\neg(Z)\rceil \theta$ and then replacing all occurrences of the form $(X) \psi$ by $(X)(\Delta(X) \supset \psi) \wedge(X)(\neg \Delta(X) \supset \psi)$. Obviously $\phi_{R}$ is logically equivalent with $\phi$. We shall abbreviate $(X)(\Delta(X) \supset \psi),(X)(\neg \Delta(X) \supset \psi)$ by $(X)+\psi$, $(X)-\psi$, respectively, and refer to $(X)^{+}$or $(X)^{-}$as positive or negative quantifiers. So in $\phi_{R}$ all quantifiers not occurring as part of subformulas $\Delta$ are positive or negative quantifiers.

By the rank of a negative quantifier $(X)^{-}$we mean the number of negative quantifiers in whose scope $(X)^{-}$stands. Let $q$ be the maximal rank of all the negative quantifiers in $\phi_{R}$.

We introduce formulas $\beta_{0}, \cdots, \beta_{q}$ such that for $0 \leqq i<q\left(\phi_{R} \wedge \beta_{i}\right)$ is true 
in a $g$-model $(G, \pi)$ if and only if $\phi_{R}$ is true in that $g$-model and there are exactly $i$ elements in $G(\iota)$ not in the trail of $\pi(K \phi)$, and $\left(\phi_{R} \wedge \beta_{q}\right)$ is true just in case $\phi_{R}$ is true and there are $q$ or more elements in $G(\iota)$ not in the trail of $\pi(K \phi) . \phi_{R}$ is of course logically equivalent to $\left(\phi_{R} \wedge \beta_{0}\right) \bigvee \cdots \vee\left(\phi_{R} \wedge \beta_{q}\right)$. So to prove the theorem it will be sufficient to find formulas $\theta_{i}$ such that $\left(\phi_{R} \wedge \beta_{i}\right)$ is logically equivalent to $\left(\theta_{i} \wedge \beta_{i}\right)$ and $\theta_{i} \alpha$-defines some class $C_{i}$, $i=0, \cdots, q$. For in that case $\left(\theta_{i} \wedge \beta_{i}\right)$ defines $C_{i}$ and $C$ must equal $C_{0} \vee \cdots$ $\vee C_{q}$, i.e., $C$ is $\alpha$-defined by $\theta_{0} \vee \cdots \vee \theta_{q}$.

First we consider the case $i=0$. Let $\theta_{0}$ be the result of replacing every occurrence of $(X)^{+} \psi \wedge(X)^{-} \psi$ in $\phi_{R}$ by $(X)^{+} \psi$. Clearly $\theta_{0} \wedge \beta_{0}$ is logically equivalent to $\phi_{R} \wedge \beta_{0}$. Since all quantifiers of $\theta_{0}$ are positive (except for those occurring inside $\Delta$ ) it is clear that $\theta_{0} \alpha$-defines some class, i.e., $\theta_{0}$ has the desired properties. This simple case provides insight into the case $i>0$.

So let $0<i \leqq q$ hold; we shall construct $\theta_{i}$. Let $(X)^{-}$be a quantifier of rank $s$, and let $\left(X_{1}\right)^{-}, \cdots,\left(X_{s}\right)^{-}$be the list of negative quantifiers with range including that of $(X)^{-}$. A $\Gamma$-formula associated with $(X)^{-}$is any of the following $s+1$ formulas: $X=X_{1}, \cdots, X=X_{s},\left(X=X \wedge X \neq X_{1} \wedge \cdots \wedge X \neq X_{s}\right)$; the index of the first $s$ of these formulas is 0 , the index of the last one is 1 . In case $(X)^{-}$has scope $\gamma \supset \psi$, where $\gamma$ is a $\Gamma$-formula for $(X)^{-}$we call $\gamma$ the restrictor of $(X)^{-}$.

Let $\phi_{-1}$ be $\phi_{R}$. For $-1 \leqq s<q$ we define $\phi_{s+1}$ as follows: if $(X)^{-}$is a quantifier of rank $s+1$ in $\phi_{s}$ with scope $\psi$ replace $(X)-\psi$ by $(X)^{-}\left(\gamma_{1} \supset \psi\right) \wedge \cdots$ $\wedge(X)^{-}\left(\gamma_{n} \supset \psi\right)$, where $\gamma_{1}, \cdots, \gamma_{n}$ is a complete set of $\Gamma$-formulas for $(X)^{-}$ except that the $\Gamma$-formula of index 1 is omitted in case the sum of the indexes of the restrictors of negative quantifiers having $(X)^{-}$in their scope is $i$. By making this replacement for all negative quantifiers of rank $s+1$ in $\phi_{s}$ one obtains $\phi_{s+1}$. Clearly $\phi_{s} \wedge \beta_{i}$ is logically equivalent to $\phi_{s+1} \wedge \beta_{i}$.

Thus we obtain the formula $\phi_{q} ; \phi_{q} \wedge \beta_{i}$ is logically equivalent to $\phi_{R} \wedge \beta_{i}$. In $\phi_{q}$ consider a negative quantifier in whose scope no negative quantifiers occur, say $(X)^{-}$with scope $\gamma \supset \psi$. Remembering the construction of $\phi_{q}$ it is simple to show that there exists a formula $\psi^{\prime}$ not containing $X$ such that the formula obtained from $\phi_{q}$ by replacing $(X)^{-}(\gamma \supset \psi)$ by $(X)^{-}\left(\gamma \supset \psi^{\prime}\right)$ is logically equivalent to $\phi_{q}$; (for in $\psi$ any primitive formula containing $X$ which is not an equality formula may be replaced by falsehood and the equality formulas $X=Y$ or $Y=X$ may be replaced by falsehood if $Y$ is bound by a positive quantifier, and by truth or falsehood as dictated by the restrictors if $Y$ is bound by a negative quantifier.) It follows that if in $\phi_{q}$ we replace $(X)^{-}(\gamma \supset \psi)$ by $(X)-\left(\neg \gamma \vee \psi^{\prime}\right)$ we obtain a formula $\phi_{q}^{\prime}$ logically equivalent to $\phi_{q}$. In $\phi_{q}^{\prime}$ replace $(X)^{-}\left(7 \gamma \vee \psi^{\prime}\right)$ by $\psi^{\prime}$, obtaining a formula $\phi_{q}^{\prime \prime}$ no longer containing the negative quantifier $(X)^{-}$. We claim that $\phi_{q}^{\prime \prime} \wedge \beta_{i}$ is equivalent to $\phi_{q}^{\prime} \wedge \beta_{i}$. To see this consider some $g$-model admissible for $\phi_{q}^{\prime}$ and some assignment $\nu$.

CASE 1. There exists a negative quantifier $(Y)^{-}$with restrictor $\gamma^{\prime}$ such that $(X)^{-}$is in the scope of $(Y)^{-}$and $\neg \Delta(Y) \wedge \gamma^{\prime}$ is made false by $\nu$. In this case 
the scope of $(Y)$ is assigned the same truth value (namely truth) by $\nu$ in both $\phi_{q}^{\prime}$ and $\phi_{q}^{\prime \prime}$. Since outside the scope of $(Y) \phi_{q}^{\prime}$ is identical with $\phi_{q}^{\prime \prime}$ both formulas obtain the same truth value. CASE 2. Case 1 fails to hold. Note that $(X)-\left(\neg \gamma \vee \psi^{\prime}\right)$ is equivalent to $(X)(\neg \Delta(X) \supset \neg \gamma) \vee \psi^{\prime}$. The construction of $\phi_{q}^{\prime}$ was designed so that if $\beta_{i}$ holds and we are in Case 2 there exists an assignment $\mu$ differing from $\nu$ at most on $X$ and making $\neg \Delta(X) \wedge \gamma$ true. So if $\nu$ makes $\beta_{i}$ true it makes $(X)(\neg \Delta(X) \supset \neg \gamma)$ false, i.e. $\nu$ assigns the same truth value to $\phi_{q}^{\prime} \wedge \beta_{i}$ and $\phi_{q}^{\prime \prime} \wedge \beta_{i}$.

Thus it is possible to eliminate successively all negative quantifiers, ending up with a formula $\theta_{i} \wedge \beta_{i}$ logically equivalent to $\phi_{R} \wedge \beta_{i}$. Clearly $\theta_{i} \alpha$-defines some class $C_{i}$, which was all that needed showing.

Using standard formulas one can generalize many results known for elementary formulas and thus widen the domain of applicability of these results. For instance the class of all structures $\pi$ with domain $\{S, P, Q\}$ such that $\pi(S), \pi(P), \pi(Q)$ represent, respectively, the sum, product and order relation of a non-archimedian field is not $\beta$-definable by any elementary set of formulas (cf. [6]) yet can easily be $\beta$-defined by a strongly standard formula. Also the class of structures $\pi$ with domain $\{S, U\}$ such that $\pi(S)$ is a group addition and $\pi(U)$ a set of operators on the group is obviously not definable by an elementary formula ( $U$ is a constant of type $((\iota))$, but can be defined by a strongly standard formula.

It is our intention merely to indicate briefly how some results known for elementary classes generalize and to mention some new problems arising in the higher order theory.

We say that the $g$-model $(G, \pi)$ is an extension of the model $\left(G^{\prime}, \pi^{\prime}\right)$ if the domain of $\pi$ includes that of $\pi^{\prime}$ and there is a one-one function $f$ mapping the domain of $G^{\prime}$ into that of $G$ in such a way that (a) $G^{\prime}(\tau)$ is mapped into $G(\tau)$ for all $\tau$, (b) $f(m)=m$ if $m \in G^{\prime}(\iota)$, (c) $f(m)\left(f\left(m_{1}\right), \cdots, f\left(m_{n}\right)\right)$ if and only if $m\left(m_{1}, \cdots, m_{n}\right)$ (d) if $a$ is in the domain of $\pi^{\prime}, f\left(\pi^{\prime}(a)\right)=\pi(a)$.

$\pi$ is an extension of $\pi^{\prime}$ or $\pi^{\prime}$ is a substructure of $\pi$ if there are $g$-models $(G, \pi)$ and $\left(G^{\prime}, \pi^{\prime}\right)$ such that the first is an extension of the second. $\pi$ is the completion of $\pi^{\prime}$ if (1) the trail of the range of $\pi$ equals the trail of the range of $\pi^{\prime}$, (2) $\pi$ is an extension of $\pi^{\prime}$, (3) the range of $\pi$ equals the trail of the range of $\pi$.

As examples of well known theorems for elementary formulas which extend at once we mention: if $\Phi$ is a set of standard formulas and $\phi$ is a standard decreasing formula such that $\Phi$ implies $\phi$, then some finite subset $\Phi^{\prime}$ of $\Phi$ implies $\phi$. (Note that our hypothesis on $\Phi$ is stronger than requiring $\Phi$ to be standard.) Also, if $\Phi$ is a standard set of formulas and $\pi$ a structure such that every substructure $\pi^{\prime}$ of $\pi$ whose completions have finite range has an extension in which $\Phi$ is true then $\pi$ has an extension in which $\Phi$ is true (cf. [3]).

Following Robinson [7] we say $\phi$ is $g$-persistent with respect to $\Phi$ if whenever $\left(G^{\prime}, \pi^{\prime}\right)$ is a $g$-model for $\phi$ and $\Phi$ and $(G, \pi)$ an extension of $\left(G^{\prime}, \pi^{\prime}\right)$ in which $\Phi$ is true then $\phi$ is true in $(G, \pi)$. The proof of [7] goes over with obvi- 
ous modifications to show that $\phi$ is $g$-persistent with respect to $\Phi$ if and only if it is logically equivalent relative to $\Phi$ to a formula in prenex form in which all initial quantifiers are existential. Let us call $\phi$ set-persistent with respect to $\Phi$ if whenever $\left(G^{\prime}, \pi^{\prime}\right)$ is a $g$-model for $\Phi \cup\{\phi\}$ and $(G, \pi)$ an extension of $\left(G^{\prime}, \pi^{\prime}\right)$ in which $\Phi$ is true and such that $G^{\prime}(\iota)=G(\iota)$ then $\phi$ is true in $(G, \pi)$. It seems possible that $\phi$ is set-persistent with respect to $\Phi$ just in case it is logically equivalent relative to $\Phi$ to a formula in prenex form in which all the higher order quantifiers are existential. In any case, this syntactic property implies set-persistence. We note that since it is easy to write down formulas that are the negations of secure formulas but which are not set-persistent it follows that not every secure formula is logically equivalent to a formula in prenex form in which all higher order quantifiers are universal. On the other hand a formula in which all terms are of order at most one is set-persistent if and only if it is strongly standard increasing.

We close with the following question: does there exist a formula $\phi$ which is both strongly standard increasing and strongly standard decreasing but which is not logically equivalent to an elementary formula? The answer is trivially yes if we permit constants of all types in $\phi$; however, if $\phi$ is to have no constants of order higher than one the problem is unsolved.

\section{BIBLIOGRAPHY}

1. L. Henkin, The completeness of the first order functional calculus, J. Symb. Logic vol. 14 (1949) pp. 159-166.

2. - Completeness in the theory of types, J. Symb. Logic vol. 15 (1950) pp. 81-91.

3. - Some interconnections between modern algebra and mathematical logic, Trans. Amer. Math. Soc. vol. 74 (1953) pp. 89-96.

4. K. J. J. Hintikka, Reductions in the theory of types, Acta Philos. Fenn. (1955) pp. 61-115.

5. A. Mostowski, On absolute properties of relations, J. Symb. Logic vol. 12 (1947) pp. 33-42.

6. A. Robinson, On the metamathematics of algebra, Amsterdam, North Holland, 1952.

7. - Note on a problem of L. Henkin, J. Symb. Logic, vol. 21 (1956) pp. 33-35.

UNIVERSITY OF MINNESOTA, Minneapolis, Minn.

UNIVERSity OF CALIFORNIA, Berkeley, Calif. 